\title{
Inhibition of Poly(I:C)-Induced Inflammation by Salvianolic Acid A in Skin Keratinocytes
}

\author{
Qing-Ling Zhang, ${ }^{1,2}$, Ri-Hua Jiang ${ }^{1}$, Xue Mei $\mathrm{Li}^{3}$, Jung-Woo Koํㄹ ${ }^{2}$, Chang Deok Kim², \\ Ming Ji Zhu ${ }^{1}$, Jeung-Hoon Lee ${ }^{2,3,4}$ \\ ${ }^{1}$ Department of Dermatology, China-Japan Union Hospital of Jilin University, Changchun, China, Departments of ${ }^{2}$ Dermatology and \\ ${ }^{3}$ Medical Science, School of Medicine, Chungnam National University, ${ }^{4}$ Skin Med Company, Daejeon, Korea
}

Background: Skin keratinocytes participate actively in inducing immune responses when external pathogens are introduced, thereby contributing to elimination of pathogens. However, in condition where the excessive inflammation is occurred, chronic skin disease such as psoriasis can be provoked. Objective: We tried to screen the putative therapeutics for inflammatory skin disease, and found that salvianolic acid A (SAA) has an inhibitory effect on keratinocyte inflammatory reaction. The aim of this study is to demonstrate the effects of SAA in poly(I:C)-induced inflammatory reaction in skin keratinocytes. Methods: We pre-treated keratinocytes with SAA then stimulated with poly(I:C). Inflammatory reaction of keratinocytes was verified using real-time polymerase chain reaction, enzyme-linked immunosorbent assay and Western blot. Results: When skin keratinocytes were pre-treated with SAA, it significantly inhibited poly (I:C)-induced expression of inflammatory cytokines including interleukin (IL)- $1 \beta$, IL-6, IL-8, tumor necrosis factor- $\alpha$,

Received July 18, 2018, Revised November 15, 2018, Accepted for publication November 17, 2018

Corresponding author: Jeung-Hoon Lee, Department of Dermatology, School of Medicine, Chungnam National University, 266 Munhwa-ro, Jung-gu, Daejeon 35015, Korea. Tel: 82-42-280-7707, Fax: 82-42-280-8459, E-mail: jhoon@cnu.ac.kr

ORCID: https://orcid.org/0000-0002-4869-940X

Ming Ji Zhu, Department of Dermatology, China-Japan Union Hospital of Jilin University, Changchun, Jilin 130033, China. Tel: 86-431-84995523, Fax: 86-431-84641026, E-mail: Zhumingji33@163.com ORCID: https://orcid.org/0000-0003-0835-2548

This is an Open Access article distributed under the terms of the Creative Commons Attribution Non-Commercial License (http://creativecommons. org/licenses/by-nc/4.0) which permits unrestricted non-commercial use, distribution, and reproduction in any medium, provided the original work is properly cited.

Copyright $\odot$ The Korean Dermatological Association and The Korean Society for Investigative Dermatology and CCL20. SAA inhibited poly(I:C)-induced activation of nuclear factor- $\kappa \mathrm{B}$ signaling. And SAA also inhibited inflammasome activation, evidenced by decrease of IL-1 $\beta$ secretion. Finally, SAA markedly inhibited poly(l:C)-induced NLRP3 expression. Conclusion: These results demonstrate that SAA has an inhibitory effect on poly(I:C)-induced inflammatory reaction of keratinocytes, suggesting that SAA can be developed for the treatment of inflammatory skin diseases such as psoriasis. (Ann Dermatol 31(3) $279 \sim 285$, 2019)

\section{-Keywords-}

Inflammasomes, Keratinocytes, Poly(I-C), Salvianolic acid A

\section{INTRODUCTION}

Skin is an organ that lies between organism and environment. It consists of three layers such as epidermis, dermis and subcutaneous fat. Skin protects organism from external stimuli such as microbes, harmful chemicals and ultraviolet light. Keratinocytes are the major cells in the epidermis that supply the fundamental protective role against harmful insults by making physical barrier. This protective barrier is produced by the sophisticated but well-ordered differentiation program of keratinocytes, in which many genes are spatiotemporally regulated to make solid waterimpermeable structure called cornified cell envelope ${ }^{1}$. In recent years, another important defensive roles of keratinocytes have been recognized. That is, keratinocytes play a role as the immune surveillance cells in innate immunity. Keratinocytes sense the signals from environmental pathogen via the toll-like receptor (TLR) system and initiate the immune responses including the increase of gene expression for many cytokines ${ }^{2,3}$. In normal condition, the 
activation of keratinocyte innate immune system helps to eliminate the external pathogens and to provoke adaptive immune response, thereby contributing to maintenance of homeostasis. However, in pathologic conditions such as psoriasis, keratinocyte activation results in abundant expression of cytokines including interleukin (IL)- $1 \beta$, tumor necrosis factor (TNF)- $\alpha$ and IL-17, thereby exacerbating the inflammatory condition and then leading to onset of disease $^{4-6}$. Considering the importance of these initial innate immune responses, the inhibition of excessive responses by keratinocytes can be a good target of drug development for inflammatory skin diseases such as psoriasis.

We attempted to screen the therapeutic materials for inflammatory skin diseases using an experimental model in which keratinocyte inflammatory reaction was induced using a double-stranded RNA (dsRNA) mimic polyinosinic: polycytidylic acid (poly $(\mathrm{l}: \mathrm{C}))^{7}$. We found that salvianolic acid $\mathrm{A}$ (SAA) has an inhibitory potential on inflammatory reaction of keratinocytes. Previously, it has been reported that SAA protects against myocardial ischemia/reperfusion injury by reducing platelet activation and inflammation ${ }^{8}$. SAA also has been reported to alleviate the ischemic brain injury through the inhibition of inflammation and apoptosis in mice 9 . However, the effects of SAA on skin keratinocytes have never been addressed so far. In this study, we provide evidence that SAA has an inhibitory effects on poly(I:C)-induced inflammatory reaction of skin keratinocytes.

\section{MATERIALS AND METHODS}

\section{Ethics statement}

Skin tissues were obtained from volunteers under the written informed consent, and the present study was approved from the ethical committee of the Institutional Review Board of Chungnam National University Hospital (IRB no. 1011-135, 2016-07-009).

\section{Cell culture and drug preparations}

The immortalized SV40T-transformed human epidermal keratinocytes (SV-HEKs) were cultured using keratinocyte-serum free medium supplemented with bovine pituitary extract (BPE) and recombinant human epidermal growth factor (rhEGF) (Life Technologies Corporation, Grand Island, NY, USA $)^{10}$. At about $70 \%$ confluency, culture medium was changed into MCDB153 (Welgene, Gyeongsan, Korea) supplemented with BPE and rhEGF. After overnight incubation, cells were treated with $1 \mu \mathrm{g} / \mathrm{ml}$ of poly(l:C) (InvivoGene, San Diego, CA, USA). SAA was purchased from Sigma-Aldrich (St. Louis, MO, USA) and dissolved in dimethyl sulfoxide (DMSO).

\section{Cytotoxicity test}

For cytotoxicity test, well-established MTT (3-(4,5-dimethyl-2-thiazolyl)-2,5-diphenyl-2H-tetrazolium bromide) assay was employed. After treatment with SAA for 24 hours, cells were incubated with MTT solution $(0.5 \mathrm{mg} / \mathrm{ml})$ and cell viability was validated by checking optical density at $570 \mathrm{~nm}$.

\section{Quantitative real-time polymerase chain reaction (qRT- PCR)}

Total RNA was purified and reverse transcribed using moloney-murine leukaemia virus reverse transcriptase (MMLVRTase; Elpis Biotech, Daejeon, Korea). The resulting cDNA was used in real-time PCR. The gene-specific primers were used as below: IL-1 $\beta$, 5'-TTAAAGCCCGCCTGACAGA and 5'-GCGAATGACAGAGGGTTTCTTAG; IL-6, 5'-CTGC GCAGCTTTAAGGAGTTC and 5'-CCATGCTACATTTGCC GAAGA; IL-8, 5'-CCTTTCCACCCCAAATTTATCA and 5'-TT TCTGTGTTGGCGCAGTGT; CCL20, 5'-CCACCTCTGCGGC GAAT and 5'-TGTGTATCCAAGACAGCAGTCAAA; TNF- $\alpha$, 5'-CTCСТTCAGACACССТСАACCT and 5'-CGACCCTAA GCCCCCAATT; GAPDH, 5'-TGCACCACCAACTGCTTAGC and 5'-GGCATGGACTGTGGTCATGAG.

\section{Enzyme-linked immunosorbent assay (ELISA)}

The secreted cytokines were quantified by ELISA. The commercial kit for IL-8 was purchased from Life Technologies Corporation. The ELISA kits for IL-1 $\beta$ and TNF- $\alpha$ were obtained from R\&D Systems (Minneapolis, MN, USA).

\section{Western blotting}

Cellular extracts were prepared using a lysis buffer (Intron, Daejeon, Korea). The cellular proteins were separated by gel electrophoresis and then transferred to nitrocellulose membranes. The membranes were incubated with primary antibody, and then sequentially incubated with peroxidase-conjugated secondary antibodies. The Western bands were obtained by enhanced chemiluminescence (Intron). The following primary antibodies were used: phospho-p65, phospho-I $\kappa \mathrm{B} \alpha$, caspase-1 (Cell Signaling Technology, Beverly, MA, USA); IL-1 $\beta$ (Abcam, Cambridge, MA, USA); NLRP3 and ASC (AdipoGen, San Diego, CA, USA); actin (Sigma-Aldrich).

\section{RESULTS}

To screen the effective materials that can inhibit poly(I:C)induced inflammation in skin keratinocytes, we initially used ELISA for IL-8 and single compounds commercially available. Among many compounds tested, SAA showed 
A<smiles>O=C(/C=C/c1ccc(O)c(O)c1/C=C/c1ccc(O)c(O)c1)OC(Cc1ccc(O)c(O)c1)C(=O)O</smiles>

B

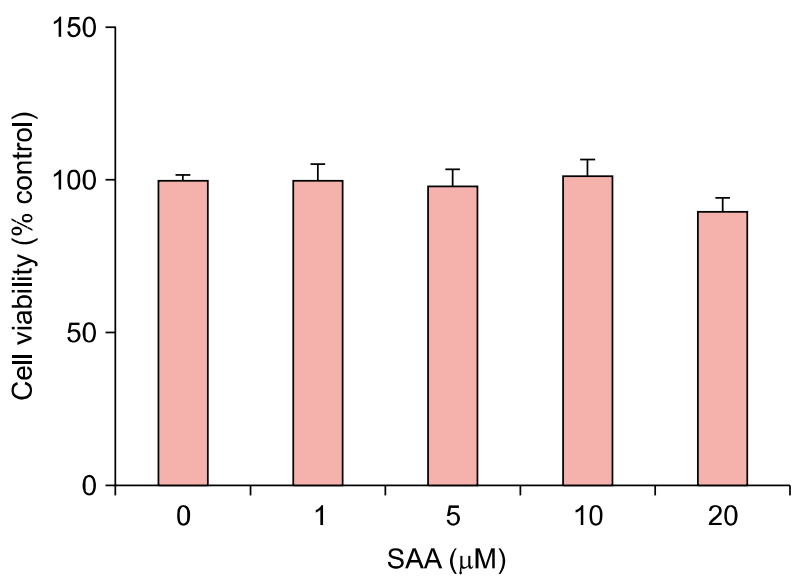

Fig. 1. (A) Structure of salvianolic acid A (SAA). (B) Cytotoxicity of SAA in keratinocytes. The immortalized SV40T-transformed human epidermal keratinocytes were treated with indicated concentrations of SAA for 24 hours. MTT assay was performed to measure cell viability. SAA did not show cytotoxicity up to $10 \mu \mathrm{M}$. The mean values \pm standard deviation are averages of triplicate measurements.

inhibitory potential on secretion of IL-8, thus we chose SAA for a further study. SAA is a polyphenolic compound (Fig. 1A), which has been originally extracted from Radix Salvia miltiorrhiza ${ }^{11}$. To determine the cytotoxicity, we treated SV-HEKs with different doses of SAA and performed MTT assay. As a result, SAA did not show the cytotoxic effect up to the dose of $10 \mu \mathrm{M}$, although insignificant slight decrease of cell viability was seen at the dose of $20 \mu \mathrm{M}$ (Fig. 1B). Therefore, we treated keratinocytes with SAA up to $10 \mu \mathrm{M}$ in the following experiments.

Poly $(\mathrm{l}: \mathrm{C})$ is a chemical mimicking the dsRNA of some viruses, which provokes innate immune reaction in a TLR3dependent fashion in skin keratinocytes ${ }^{12}$. To examine the effect of SAA, we pre-treated SV-HEKs with SAA then stimulated with poly(l:C). Inflammatory reaction was first determined by qRT-PCR. In the absence of pre-treatment with SAA, poly(I:C) resulted in dramatic increase of mRNAs for inflammatory cytokines including IL-1 $\beta$, IL-6, IL-8, TNF- $\alpha$, and CCL20. By contrast, pre-treatment of SAA significantly inhibited poly(I:C)-induced expression of inflammatory cytokines (Fig. 2A). We next checked the secreted cytokines from keratinocytes using ELISA. Consistent with the data obtained from qRT-PCR, poly $(I: C)$ treatment led to dramatic increase of secretion of inflammatory cytokines such as IL-1 $\beta$, IL-8, and TNF- $\alpha$ from keratinocytes. Pre-treatment with SAA markedly inhibited poly(l:C)-induced secretion of inflammatory cytokines (Fig. 2B).

It has been well recognized that nuclear factor- $\kappa \mathrm{B}$ (NF- $\kappa \mathrm{B})$ signaling is pivotal in innate immune responses of skin keratinocytes ${ }^{13,14}$. To delineate the potential mechanism, we investigated the effect of SAA on activation of NF- $\kappa$ B signaling. Consistent with previous data, poly $(\mathrm{l}: \mathrm{C})$ treat- ment led to activation of NF- $\kappa$ B signaling that was evidenced by increase of phosphorylation of p65 subunit and I $\kappa \mathrm{B} \alpha$. Pre-treatment of keratinocytes with SAA resulted in significant inhibition of poly(l:C)-induced phosphorylation of p65 and I $\kappa \mathrm{B} \alpha$ (Fig. 3A). In addition to NF- $\kappa \mathrm{B}$ signaling, mitogen-activated protein kinases (MAPKs) are also implicated in poly(I:C)-induced inflammatory reaction in keratinocytes and other systems ${ }^{15-17}$. Thus, we examined whether SAA can also inhibit MAPK pathway using Western blot. As a result, SAA significantly inhibited poly (I:C)-induced phosphorylation of p38 MAPK, JNK, and ERK1/2 (Fig. 3B).

When skin keratinocytes were stimulated by poly $(\mathrm{l} ; \mathrm{C})$, the large caspase-1-activating complex called inflammasome is assembled and then proteolytic activation of IL-1 $\beta$ is occurred. The active IL-1 $\beta$ is then released and participates in the induction of inflammation, contributing to establishment of inflammatory skin diseases such as psoriasis $^{7,18}$. To examine whether SAA affects inflammasome activation, we collected cell culture medium and then checked the released IL-1 $\beta$ and caspase-1. As anticipated, treatment of keratinocytes with poly $(\mathrm{I}: \mathrm{C})$ resulted in remarkable increase of IL-1 $\beta$ secretion and inflammasomeactivated caspase-1. Pre-treatment with SAA markedly inhibited the secretion of IL-1 $\beta$ and caspase-1 (Fig. 4A). As the inflammasome is a complex structure in which component proteins such as NLRP3 and ASC are involved ${ }^{19}$, we next examined the protein levels of NLRP3 and ASC. Poly $(\mathrm{I}: \mathrm{C})$ increased the protein level of NLRP3, and this poly(l:C)-increased NLRP3 was obviously inhibited by SAA. However, there was no significant effect on ASC level by poly(I:C) treatment (Fig. 4B). 
A
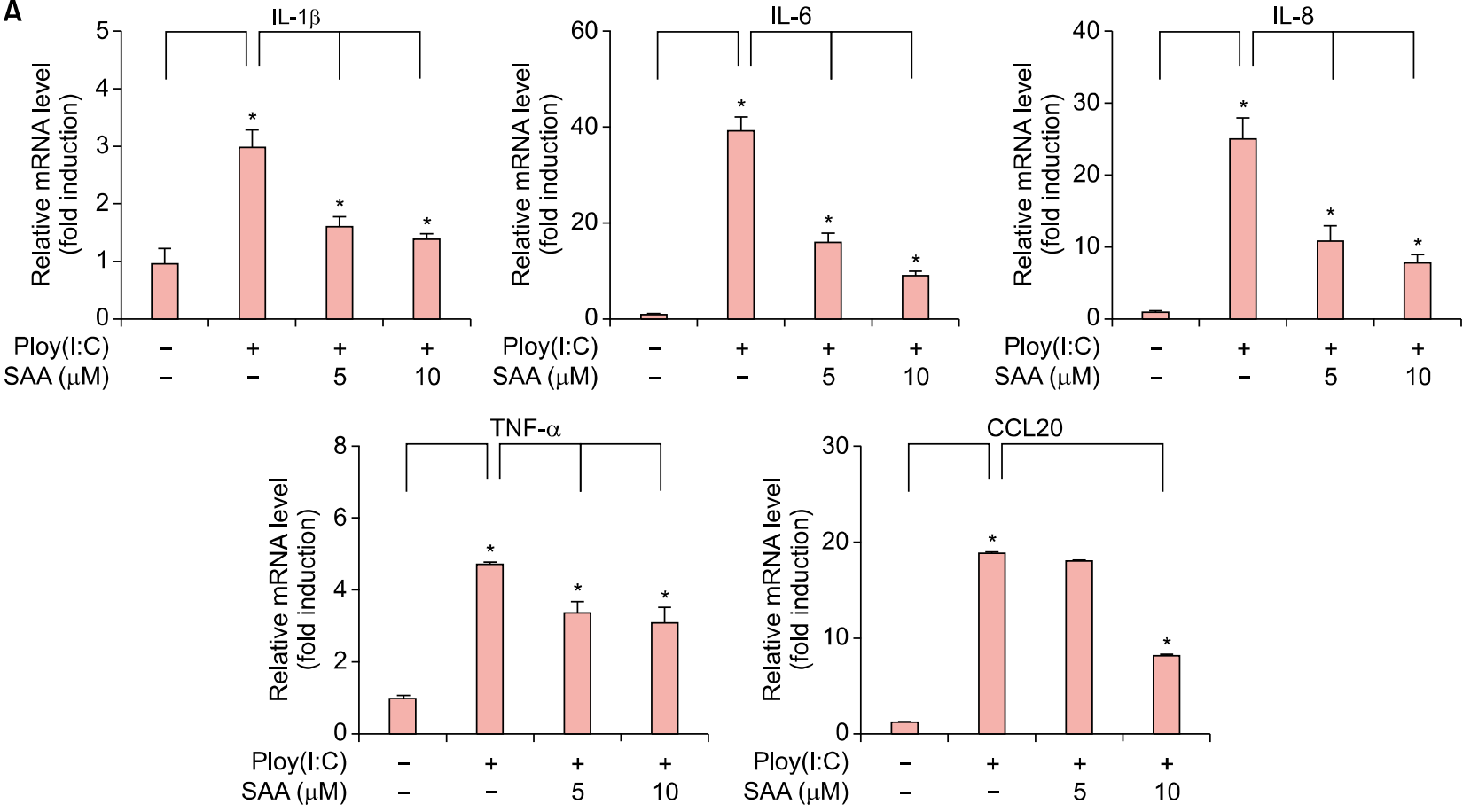

B
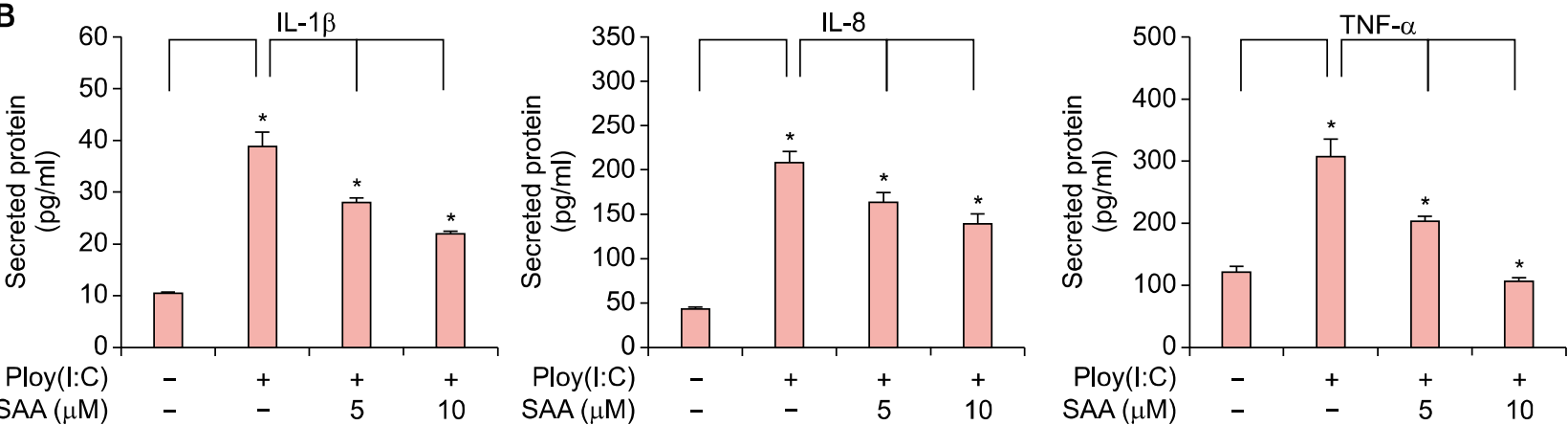

Fig. 2. Effect of salvianolic acid $A(S A A)$ on poly(I:C)-induced inflammatory reaction in keratinocytes. (A) The immortalized SV40T-transformed human epidermal keratinocytes (SV-HEKs) were pre-treated with SAA at the indicated concentrations for 1 hour, and then stimulated with $1 \mu \mathrm{g} / \mathrm{ml}$ of poly(I:C) for 2 hours. The mRNA level was assessed by quantitative real-time polymerase chain reaction. SAA inhibited poly(l:C)-induced cytokine expressions. Data are expressed as fold induction. (B) SV-HEKs were pre-treated with SAA at the indicated concentrations for 1 hour, and then stimulated with $1 \mu \mathrm{g} / \mathrm{ml}$ of poly(l:C) for 24 hours. Secretion of cytokines was measured by enzyme-linked immunosorbent assay. SAA inhibited poly(I:C)-induced secretion of cytokines from SV-HEKs. The mean values \pm standard deviation are averages of triplicate measurements. IL: interleukin, TNF- $\alpha$ : tumor necrosis factor- $\alpha .{ }^{*} p<0.01$.

\section{DISCUSSION}

Skin keratinocytes play an important role in protection of organism by providing physical and functional defense systems. The one involves barrier structure that is composed of water-insoluble corneocytes and intercellular lipid molecules, the other includes immune modulatory potential in which many cytokines and chemokines are coordinately regulated by pathogen-sensing machinery. Especially for innate immunity, keratinocytes exert their important role as a primary guardian against non-self and/or self antigens through the activation of TLR systems. It has been demonstrated that keratinocytes express a line of functional TLRs including TLR3, 4, 5, 7 and 9 $9^{20,21}$. Among them, TLR3 acts as a receptor for dsRNA that can be found in some viruses. In condition related to skin disease such as psoriasis, keratinocytes are susceptible to viral RNA intermediates, and activation of TLR3 leads to drastic increase of pro-inflammatory cytokines and chemokines including IL- $1 \beta$, TNF- $\alpha$, IL- $6, \mathrm{IL}-8$ and $\mathrm{CCL} 20^{22}$. The increased inflammatory cytokines in turn contributes to recruit and activate immune cells such as neutrophils and 
A

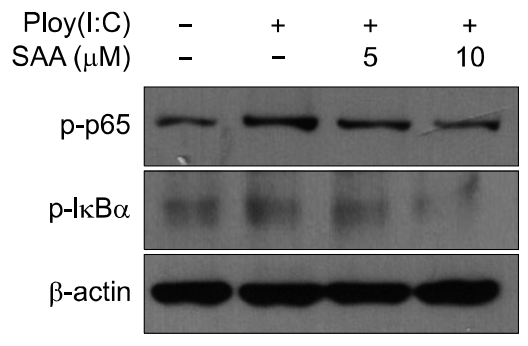

B

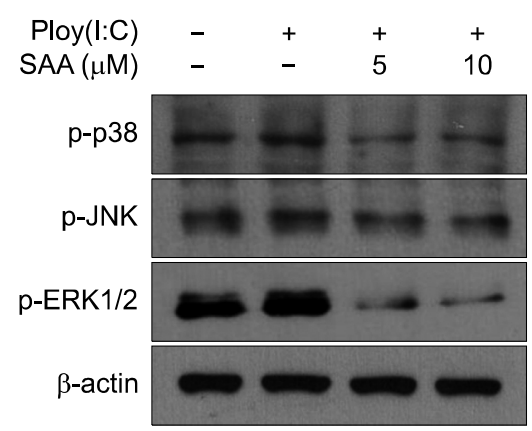

Fig. 3. Effect of salvianolic acid $A(S A A)$ on poly(l:C)-induced nuclear factor- $\kappa$ B (NF- $\kappa$ B) activation in keratinocytes. (A) The immortalized SV40T-transformed human epidermal keratinocytes were pre-treated with SAA at the indicated concentrations for 1 hour, and then stimulated with $1 \mu \mathrm{g} / \mathrm{ml}$ of poly(l:C) for 1 hour. The protein levels of phosphorylated-p65 (p-p65) and phosphorylated-I $\kappa \mathrm{B} \alpha$ $(\mathrm{p}-\mathrm{l} \kappa \mathrm{B} \alpha)$ were determined by Western blot. SAA inhibited poly(l:C)-induced activation of $\mathrm{NF}-\kappa \mathrm{B}$. $\beta$-actin was used for internal control. (B) Effect of SAA on phosphorylation of mitogen-activated protein kinases (MAPKs). SAA inhibited poly(l:C)-induced phosphorylation of p38 MAPK, JNK, and ERK1/2.

A

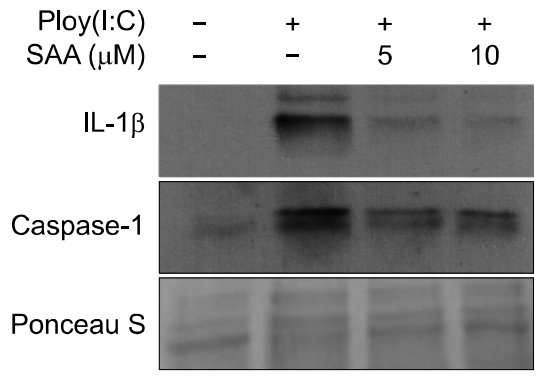

B

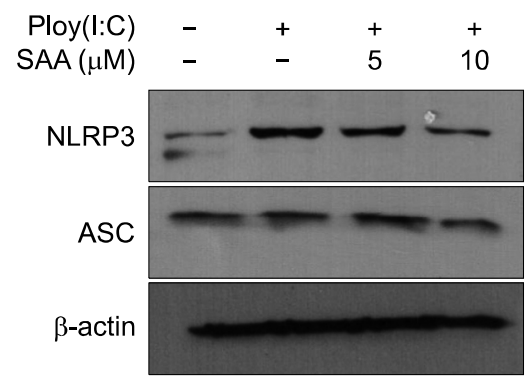

Fig. 4. Effect of salvianolic acid $A(S A A)$ on poly(I:C)-induced inflammasome activation in keratinocytes. (A) The immortalized SV40T-transformed human epidermal keratinocytes were pre-treated with SAA at the indicated concentrations for 1 hour, and then stimulated with $1 \mu \mathrm{g} / \mathrm{ml}$ poly(l:C) for 24 hours. Culture medium was collected and concentrated, then subjected to Western blot to assess the secreted protein levels for interleukin (IL)-1 $\beta$ and caspase-1. SAA inhibited poly(I:C)-induced secretion of IL-1 $\beta$ and caspase-1. Ponceau S staining was used for loading control. (B) After treatment with SAA, cellular extracts were prepared. The protein levels of NLRP3 and ASC were determined by Western blot. SAA inhibited poly(l:C)-induced NLRP3 expression. $\beta$-actin was used for internal control.

activated $\mathrm{T}$ cells ${ }^{23}$. Thus, the drug screening against inflammatory reaction of keratinocytes is a good approach for the development of therapeutics for skin diseases such as psoriasis. In this study, we provide evidence that SAA inhibits poly(I:C)-induced inflammatory reaction of keratinocytes, suggesting that SAA can be applicable for the treatment of skin disease such as psoriasis.

$\mathrm{SAA}$ is a polyphenolic depside that is composed of three of monocyclic aromatic units linked by ester bond (Fig. 1A). It was originally isolated from the dried roots of Salvia miltiorrhiza $^{11}$. SAA has a variety of biological and pharmacological activities, including anti-tumor, anti-metastatic, antioxidant, and anti-inflammatory effects. For example, SAA induces cell apoptosis and suppresses tumor growth in acute myeloid leukemia via the inhibition of PI3K/Akt sig- naling ${ }^{24}$. Other evidence indicates that SAA inhibits the migration and invasion of human breast cancer cells by inactivating transgelin $2^{25}$. In addition, SAA inhibits the highfat diet-induced hepatic inflammation via a NLRP3 inflammasome-dependent way in rat liver ${ }^{26}$.

In this study, we demonstrated that SAA has inhibitory potential on poly(I:C)-induced inflammatory reaction in skin keratinocytes. The effects of SAA were likely due to its inhibitory potential on NF- $\kappa$ B signaling, because that NF- $\kappa$ $B$ is key player in the inflammatory reaction. As well recognized, NF- $\kappa$ B directly binds to promoter of many inflammatory cytokine genes, thereby provoking consequent relay of inflammatory process. It has been reported that SAA attenuates NF- $\kappa$ B activation in other systems such as mouse macrophages and rat renal tissues ${ }^{27,28}$. Thus, it is 
plausible that the SAA inhibits the activation of NF- $\kappa$ B in skin keratinocytes thereby affecting downstream cellular events. In this regard, it is likely that the inhibition of inflammasome activation by SAA is also linked to NF- $\kappa \mathrm{B}$ signaling. Inflammasome activation is an important process in innate immunity, and it has been also recognized that NF- $\kappa$ B activation plays a role in inflammasome activation. When pathogen-associated molecular patterns or damage-associated molecular patterns are recognized by defense cells, inflammasome component protein NLRP3 associates with the adaptor protein ASC. The assembly of NLRP3 and ASC results in the formation of protein complex that can activate the caspase- 1 by proteolytic cleavage of pro-caspase- $1^{29}$. The resultant active caspase- 1 is then secreted from keratinocytes, thereby contributing to the pathogenesis of skin disease such as psoriasis ${ }^{30}$. In our study, SAA markedly inhibited poly(l:C)-induced activation of NF- $\kappa$ B signaling, and also inhibited inflammasome activation in terms of decreasing the NLRP3 level and the IL-1 $\beta$ secretion. Thus, our data provide evidence supporting that SAA can be used for treating innate immunity-related skin diseases.

In summary, we demonstrated that SAA inhibited poly (I:C)-induced inflammatory reaction of epidermal keratinocytes. Our data suggest that SAA would be a candidate for treatment of chronic inflammatory skin disease such as psoriasis.

\section{ACKNOWLEDGMENT}

This study was supported by a grant from the National Research Foundation of Korea (NRF-2015R1D1A1A090 58799). This study was also supported by Technological Development Plan Project in Jilin Province of China (20170414056GH).

\section{CONFLICTS OF INTEREST}

The authors have nothing to disclose.

\section{ORCID}

Qing-Ling Zhang, https://orcid.org/0000-0002-0866-1140 Ri-Hua Jiang, https://orcid.org/0000-0002-7957-9427 Xue Mei Li, https://orcid.org/0000-0002-1231-2891 Jung-Woo Ko, https://orcid.org/0000-0002-3479-6305 Chang Deok Kim, https://orcid.org/0000-0001-9341-6491 Ming Ji Zhu, https://orcid.org/0000-0003-0835-2548 Jeung-Hoon Lee, https://orcid.org/0000-0002-4869-940X

\section{REFERENCES}

1. Nemes Z, Steinert PM. Bricks and mortar of the epidermal barrier. Exp Mol Med 1999;31:5-19.

2. Trinchieri G, Sher A. Cooperation of Toll-like receptor signals in innate immune defence. Nat Rev Immunol 2007;7: 179-190.

3. Greb JE, Goldminz AM, Elder JT, Lebwohl MG, Gladman DD, Wu JJ, et al. Psoriasis. Nat Rev Dis Primers 2016;2:16082.

4. Hawkes JE, Chan TC, Krueger JG. Psoriasis pathogenesis and the development of novel targeted immune therapies. J Allergy Clin Immunol 2017;140:645-653.

5. Kim J, Krueger JG. The immunopathogenesis of psoriasis. Dermatol Clin 2015;33:13-23.

6. Li ZJ, Choi DK, Sohn KC, Lim SK, Im M, Lee $Y$, et al. Induction of Interleukin-22 (IL-22) production in CD4(+) T cells by IL-17A secreted from CpG-stimulated keratinocytes. Ann Dermatol 2016;28:579-585.

7. Grimstad $\varnothing$, Husebye $H$, Espevik T. TLR3 mediates release of IL- $1 \beta$ and cell death in keratinocytes in a caspase-4 dependent manner. J Dermatol Sci 2013;72:45-53.

8. Yuan X, Xiang Y, Zhu N, Zhao X, Ye S, Zhong P, et al. Salvianolic acid A protects against myocardial ischemia/ reperfusion injury by reducing platelet activation and inflammation. Exp Ther Med 2017;14:961-966.

9. Chien MY, Chuang $\mathrm{CH}$, Chern CM, Liou KT, Liu DZ, Hou $\mathrm{YC}$, et al. Salvianolic acid A alleviates ischemic brain injury through the inhibition of inflammation and apoptosis and the promotion of neurogenesis in mice. Free Radic Biol Med 2016;99:508-519.

10. Choi HI, Sohn KC, Hong DK, Lee Y, Kim CD, Yoon TJ, et al. Melanosome uptake is associated with the proliferation and differentiation of keratinocytes. Arch Dermatol Res 2014;306: 59-66.

11. Lian-Niang L, Rui T, Wei-Ming C. Salvianolic acid A, a new depside from roots of salvia miltiorrhiza. Planta Med 1984; 50:227-228.

12. Rana AA, Lucs AV, DeVoti J, Blanc L, Papoin J, Wu R, et al. Poly(I:C) induces controlled release of IL-36 $\gamma$ from keratinocytes in the absence of cell death. Immunol Res 2015; 63:228-235.

13. Vu AT, Chen X, Xie Y, Kamijo S, Ushio H, Kawasaki J, et al. Extracellular double-stranded RNA induces TSLP via an endosomal acidification- and NF- $\kappa \mathrm{B}$-dependent pathway in human keratinocytes. J Invest Dermatol 2011;131:22052212.

14. Sohn KC, Back SJ, Choi DK, Shin JM, Kim SJ, Im M, et al. The inhibitory effect of A20 on the inflammatory reaction of epidermal keratinocytes. Int J Mol Med 2016;37:1099-1104.

15. Rudraiah S, Shamilov R, Aneskievich BJ. TNIP1 reduction sensitizes keratinocytes to post-receptor signalling following exposure to TLR agonists. Cell Signal 2018;45:81-92.

16. Zhu S, Xu X, Liu K, Gu Q, Wei F, Jin H. Peptide GC31 inhibits chemokines and ICAM- 1 expression in corneal fibroblasts exposed to LPS or poly $(\mathrm{I}: \mathrm{C})$ by blocking the NF- $\kappa$ B and MAPK pathways. Exp Eye Res 2017;164:109-117.

17. Fischetti L, Zhong Z, Pinder CL, Tregoning JS, Shattock RJ. 
The synergistic effects of combining TLR ligand based adjuvants on the cytokine response are dependent upon p38/JNK signalling. Cytokine 2017;99:287-296.

18. Sun $\mathrm{Y}$, Zhang J, Zhai T, Li H, Li H, Huo R, et al. CCN1 promotes IL-1 $\beta$ production in keratinocytes by activating p38 MAPK signaling in psoriasis. Sci Rep 2017;7:43310.

19. Muruve DA, Pétrilli V, Zaiss AK, White LR, Clark SA, Ross $\mathrm{PJ}$, et al. The inflammasome recognizes cytosolic microbial and host DNA and triggers an innate immune response. Nature 2008;452:103-107.

20. Lebre MC, van der Aar AM, van Baarsen L, van Capel TM, Schuitemaker JH, Kapsenberg ML, et al. Human keratinocytes express functional Toll-like receptor 3, 4, 5, and 9. J Invest Dermatol 2007;127:331-341.

21. Li ZJ, Sohn KC, Choi DK, Shi G, Hong D, Lee HE, et al. Roles of TLR7 in activation of NF- $\kappa \mathrm{B}$ signaling of keratinocytes by imiquimod. PLoS One 2013;8:e77159.

22. Grimstad O, Pukstad B, Stenvik J, Espevik T. Oligodeoxynucleotides inhibit Toll-like receptor 3 mediated cytotoxicity and CXCL8 release in keratinocytes. Exp Dermatol 2012;21:7-12.

23. Sweeney CM, Tobin AM, Kirby B. Innate immunity in the pathogenesis of psoriasis. Arch Dermatol Res 2011;303: 691-705.

24. Pei R, Si T, Lu Y, Zhou JX, Jiang L. Salvianolic acid A, a novel PI3K/Akt inhibitor, induces cell apoptosis and sup- presses tumor growth in acute myeloid leukemia. Leuk Lymphoma 2018;59:1959-1967.

25. Zheng X, Chen S, Yang Q, Cai J, Zhang W, You H, et al. Salvianolic acid $A$ reverses the paclitaxel resistance and inhibits the migration and invasion abilities of human breast cancer cells by inactivating transgelin 2. Cancer Biol Ther 2015;16:1407-1414.

26. Ding C, Zhao Y, Shi X, Zhang N, Zu G, Li Z, et al. New insights into salvianolic acid $A$ action: regulation of the TXNIP/NLRP3 and TXNIP/ChREBP pathways ameliorates HFD-induced NAFLD in rats. Sci Rep 2016;6:28734.

27. Li L, Xu T, Du Y, Pan D, Wu W, Zhu H, et al. Salvianolic acid $A$ attenuates cell apoptosis, oxidative stress, Akt and NF- $\mathrm{KB}$ activation in angiotensin-II induced murine peritoneal macrophages. Curr Pharm Biotechnol 2016;17:283-290.

28. Fan HY, Yang MY, Qi D, Zhang ZK, Zhu L, Shang-Guan $X X$, et al. Salvianolic acid $A$ as a multifunctional agent ameliorates doxorubicin-induced nephropathy in rats. Sci Rep 2015;5:12273.

29. Elliott El, Sutterwala FS. Initiation and perpetuation of NLRP3 inflammasome activation and assembly. Immunol Rev 2015; 265:35-52.

30. Carlström M, Ekman AK, Petersson S, Söderkvist P, Enerbäck C. Genetic support for the role of the NLRP3 inflammasome in psoriasis susceptibility. Exp Dermatol 2012;21:932-937. 